PROCEEDINGS OF THE

AMERICAN MATHEMATICAL SOCIETY

Volume 127, Number 3, March 1999, Pages 725-735

S 0002-9939(99)04864-9

\title{
A ONE-POINT ATTRACTOR THEORY FOR THE NAVIER-STOKES EQUATION ON THIN DOMAINS WITH NO-SLIP BOUNDARY CONDITIONS
}

\author{
JOEL D. AVRIN
}

(Communicated by Jeffrey B. Rauch)

\begin{abstract}
In an earlier paper related to recent results of Raugel and Sell for periodic boundary conditions, we considered the incompressible Navier-Stokes equations on 3-dimensional thin domains with zero ("no-slip") boundary conditions and established global regularity results. We extend those results here by developing an attractor theory. We first show that under similar thinness restrictions trajectories of solutions approach each other in $L^{4}$-norm exponentially. Next, for constant-in-time forcing data $f_{1}=f_{1}(x)$, we suppose that $f(t) \rightarrow f_{1}$ in $L^{2}$ as $t \rightarrow+\infty$, and show that if $v$ and $w_{1}$ solve the equations with forcing data $f$ and $f_{1}$, respectively, then $\left\|v(t)-w_{1}(t)\right\|_{4} \rightarrow 0$ as $t \rightarrow+\infty$.

For similar thinness restrictions we show that the steady-flow equations with forcing data $f_{1}$ have a unique solution $u_{s}$. Under both thinness assumptions we then have that all solutions $v(t)$ converge to $u_{s}$ in $L_{4}$ as $t \rightarrow+\infty$; thus we have a one-point attractor for strong solutions. In fact, we have a onepoint attractor for the Leray solutions as well. Moreover, under significantly more relaxed thinness assumptions we are able to show that Leray solutions nonetheless eventually become regular.
\end{abstract}

\section{INTRODUCTION}

We consider the incompressible Navier-Stokes equations

$$
\begin{gathered}
u_{t}+(u \cdot \nabla) u-\nu \Delta u+\nabla p=g, \\
\nabla \cdot u=0
\end{gathered}
$$

over a bounded domain $\Omega \subset \Re^{3}$ with smooth boundary $\partial \Omega$. Here $u=\left(u_{1}, u_{2}, u_{3}\right)$ denotes the fluid velocity, $p$ denotes the pressure, $g=\left(g_{1}, g_{2}, g_{3}\right)$ is the density per unit volume, and $\nu=1 / R e$, where $R e$ is the Reynolds number. We have that $u=u(x, t), p=p(x, t)$, and $g=g(x, t)$, where $(x, t) \in \Omega \times[0,+\infty)$.

We will use $L^{p}(\Omega)$ and $\left(L^{p}(\Omega)\right)^{n}$ interchangeably in what follows as it will be clear from the context when we are taking $L^{p}$-norms component-wise. With this in mind we reformulate (1.1) in standard fashion as a Cauchy problem on $H=L^{2}(\Omega)$ : let $-\Delta$ be equipped with zero Dirichlet boundary conditions, let $H_{\sigma}$ be the closure in $H$ of $\left\{h \in C_{0}^{\infty}(\Omega) \mid \nabla \cdot h=0\right\}$, and let $P$ be the projection onto $H_{\sigma}$, i.e. $P H=H_{\sigma}$. For simplicity we set $\nu=1$; in our concluding remarks we will then

Received by the editors August 7, 1996 and, in revised form, June 9, 1997.

1991 Mathematics Subject Classification. Primary 35B40, 35Q10.

(C)1999 American Mathematical Society 
indicate how our results generalize to the case of arbitrary Reynolds number. With this in mind let $A=-P \Delta$ be the Stokes operator and let $f=P g$; then taking $u_{0} \in H_{\sigma}$ and applying $P$ to both sides of (1.1a) we rewrite (1.1) as

$$
\begin{gathered}
u_{t}=-A u-P(u \cdot \nabla) u+f, \\
u(x, 0)=u_{0}(x) .
\end{gathered}
$$

The study of this problem has a vast and well-known history; see e.g. [1], [2], [4], [5], [6], [7], [8], [9], [10], [11], [12], [13], [14] and the references contained therein. For our purposes here we briefly summarize the development of the existence and uniqueness theory for strong solutions of (1.2): for suitable $u_{0}$ and $f$ unique local strong solutions are known to exist in dimensions $n=2$ and $n=3$, and these solutions are known to be global if $n=2$. For $n=3$ global strong solutions have until recently only been known to exist for small initial data.

In [11] Raugel and Sell treated (1.2) on thin domains: let $\Omega=\Omega^{\prime} \times(0, \varepsilon)$, where $\Omega^{\prime}$ is a domain in $\Re^{2}$. Focussing primarily on the case of periodic boundary conditions (in which case $\Omega^{\prime}$ is a rectangle), their results on the existence and uniqueness of global strong solutions allow $\left\|A^{1 / 2} u_{0}\right\|_{2}$ to be large in proportion to a fractional negative power of $\varepsilon$. Raugel and Sell also establish the existence of a unique local maximal attractor for which the strong solutions they establish are in the basin of attraction; moreover this attractor globally attracts the Leray (weak) solutions. They also show that the trajectories of their global solutions asymptotically approximate the flow on the 2-dimensional attractor (as constructed in e.g. [2]) for small enough $\varepsilon$. While partial results on global strong solutions for other boundary conditions are also obtained, again we note that the primary focus in [11] is on the case of periodic boundary conditions.

In [1] we focused on the case of zero Dirichlet boundary conditions (as will be done in this paper). This case is quite distinct from the periodic case, in that on thin domains the first eigenvalue of $-\Delta$ is large. In particular let $\Omega=\Omega^{\prime} \times(0, \varepsilon)$ for an arbitrary domain $\Omega^{\prime}$ in $\Re^{2}$. Let $0<\lambda_{1}^{\prime}<\lambda_{2}^{\prime} \ldots$ be the eigenvalues for the 2 -dimensional version of $-\Delta$ on $\Omega^{\prime}$; then by separation of variables the eigenvalues for $-\Delta$ on $\Omega$ are $\lambda_{m n}=\lambda_{m}+(n \pi / \varepsilon)^{2}$ for $m, n \geq 1$. Let $\lambda_{1}$ denote the smallest eigenvalue; then $\lambda_{1}=\left(\varepsilon^{2} \lambda_{1}^{\prime}+\pi^{2}\right) / \varepsilon^{2} \rightarrow+\infty$ as $\varepsilon \rightarrow 0$. This property of $\lambda_{1}$, plus the fact that $\lambda_{1}$ is a lower bound on the first eigenvalue of $A$, was observed in [1] to be quite useful in the construction of global strong solutions that, as in [11], can have large initial data. Since in fact the analysis in [1] only depends on the size of $\lambda_{1}$, more general thin domains can be considered; we simply define a domain to be generalized thin in $\Re^{3}$ if the first eigenvalue of $-\Delta$ equipped with zero Dirichlet boundary conditions is large.

For a parameter $M$ depending on $\left\|u_{0}\right\|_{4}$ and various norms on $f$, the analysis in [1] used a contraction mapping principle to establish the existence and uniqueness of global strong solutions of (1.2) whenever $\lambda^{-1 / 8} M<1 / K_{1}$. Here $K_{1}$ is a constant depending e.g. on a constant appearing in a Sobolev inequality and on a constant expressing the analyticity of $\exp (-t A)$. It was seen in [1] that $K_{1}$ can be chosen so as not to increase as $\lambda_{1}$ increases. Thus $M$ can be arbitrarily large provided that $\lambda_{1}$ is large enough.

In this way the study of the existence and uniqueness of global regular solutions with large initial data on thin domains, as initiated by Raugel and Sell, was thus extended in [1] to the case of zero Dirichlet boundary conditions. Moreover, by 
focusing on $\lambda_{1}$, the study was extended to a very general class of thin domains. We would now like to continue to extend the Raugel/Sell program in this setting by developing an attractor theory. Since bounds on all derivatives of $u$ were established in [1], it is easy to establish the existence of a unique local maximal attractor $\mathcal{A}$ for which the strong solutions constructed in [1] are in the basin of attraction; indeed, the proof is very standard and can safely be omitted. With a little more work we can show that $\mathcal{A}$ globally attracts the Leray solutions. We do this by a fairly straightforward calculation in section 2 below (see (2.6)-(2.16)). In the discussion that follows (2.18), in particular, we will see that we have such a global attractor in a surprisingly large number of cases.

The main purpose of this paper, however, is to show that when further size restrictions are placed on $\lambda_{1}$ beyond those assumed in [1] (but not drastically so, by any means), then the attractor $\mathcal{A}$ consists of a simple point. Let

$$
L \equiv \sup _{t \geq 0}\|f(t)\|_{2}^{2},
$$

where here and in what follows we replace $f(x, t)$ by $f(t)$ when it is clear from the context that we are dealing with a function of $t$ into a Banach space. For any $\delta>0$ we will first show (in section 2) that if $u$ is any Leray solution of (1.2) then there exists a $\tau_{u}>0$ such that

$$
\left\|A^{1 / 2} u(t)\right\|_{2}^{2} \leq(1+\delta) \lambda_{1}^{-1 / 2} L
$$

for all $t \geq \tau_{u}$. Our first theorem applies to any two strong solutions satisfying (1.4). Its relationship with the strong solutions constructed in [1] and with the Leray solutions will be discussed following the statement of the theorem.

Theorem 1.1. Let $v$ and $w$ be two strong solutions of (1.2) satisfying (1.4). Let $\gamma_{6}=\lambda_{1} / 6$. Then if $\lambda_{1}$ is large enough there exists a constant $K_{0}$ depending only on $\Omega$ and $A$ such that

$$
\|v(t)-w(t)\|_{4} \leq K_{0} e^{-\gamma_{6} t}
$$

for all $t \geq 0$.

We will prove this theorem in section 3 below. Meanwhile if $v$ and $w$ are two Leray solutions, set $\tau_{v w}=\max \left\{\tau_{v}, \tau_{w}\right\}$. It will be shown in section 2 using (1.4) that if $\lambda_{1}$ is large enough, then $\left\|v\left(\tau_{v w}\right)\right\|_{4}$ and $\left\|w\left(\tau_{v w}\right)\right\|_{4}$ satisfy the size restrictions imposed on initial data in [1] that guarantee the existence and uniqueness of global strong solutions of (1.2). Since it is known that strong solutions are unique in the class of weak solutions, we see that $v(t)$ and $w(t)$ continue as strong solutions of (1.2) satisfying (1.4) for all $t \geq \tau_{v w}$. Combining these remarks with Theorem 1.1 we thus obtain the following result:

Corollary 1.2. Let $v$ and $w$ be any two Leray solutions of (1.2) with $\tau_{v w}$ as above. Then if $\lambda_{1}$ is large enough we have that

$$
\|v(t)-w(t)\|_{4} \leq K_{0} e^{-\gamma_{6}\left(t-\tau_{v w}\right)}
$$

for all $t \geq \tau_{v w}$, where $K_{0}$ and $\gamma_{6}$ are as in Theorem 1.1 .

Thus if $\lambda_{1}$ is large enough all solutions asymptotically approach a single trajectory as $t \rightarrow+\infty$. We now suppose that there exists an $f_{1}=f_{1}(x)$ such that

$$
\lim _{t \rightarrow+\infty}\left\|f(t)-f_{1}\right\|_{2}=0 .
$$


The next result will be proven in section 3 as a corollary of the proofs of Theorem 1.1 and Corollary 1.2:

Theorem 1.3. Suppose that $w_{1}$ is a strong solution of (1.2) satisfying (1.4) with $f$ replaced by $f_{1}$. For any given $f$ such that (1.7) holds, let $v$ be a Leray solution of (1.2). Then if $\lambda_{1}$ is large enough we have that

$$
\lim _{t \rightarrow+\infty}\left\|v(t)-w_{1}(t)\right\|_{4}=0 .
$$

In stating this last result we anticipate showing that $w_{1}(t)$ converges to a steadyflow solution as $t \rightarrow+\infty$. In fact it does, and this steady-flow solution is unique, provided that $\lambda_{1}$ is large enough. The remarks that follow will establish both facts from the above developments and the next result.

Theorem 1.4. Let $f_{1} \in H_{\sigma}$. Then if $\lambda_{1}$ is large enough the steady-flow problem

$$
A u+P(u \cdot \nabla) u=f_{1}
$$

has a unique solution $u_{s}$ satisfying

$$
\left\|A^{1 / 2} u_{s}\right\|_{2}^{2} \leq\left\|\lambda_{1}^{-1 / 2} f_{1}\right\|_{2}^{2}
$$

We now remark that if $\lambda_{1}$ is large enough so that both Theorem 1.1 and Theorem 1.4 hold, then since the $u_{s}$ of Theorem 1.4 clearly satisfies (1.4) by virtue of (1.10), we have that $w_{1}$ and $u_{s}$ satisfy the estimate (1.5), thus establishing the convergence of $w_{1}$ to a unique steady-flow solution. Combining this last observation with Theorem 1.4, we thus obtain the following one-point attractor result for Leray solutions on domains that are sufficiently generalized thin:

Theorem 1.5. For any given $f$ such that (1.7) holds, let $v$ be a Leray solution of (1.2). Then if $\lambda_{1}$ is large enough so that Theorems 1.3 and 1.4 hold, then for the unique solution $u_{s}$ of (1.9) we have that

$$
\lim _{t \rightarrow+\infty}\left\|v(t)-u_{s}\right\|_{4}=0 .
$$

It remains to prove Theorems 1.1 and 1.4, and establish the estimate (1.4). This will be done in sections 3 and 2, respectively. Section 2 will also contain some useful preliminary observations and remarks.

\section{Preliminary observations}

We first observe the well-known fact, following from a simple inner product argument, that for all $v \in D(A)$

$$
\left\|A^{1 / 2} v\right\|_{2}=\|\nabla v\|_{2} .
$$

Next we set $X_{p}=P L^{p}(\Omega)$ and note that by [5, Proposition 1.4] $D\left(A^{\alpha / 2}\right)$ is continuously embedded in $X_{2} \cap H^{\alpha}(\Omega)$ for all $\alpha \geq 0$. From this and the Sobolev embedding theorems it follows, as in the proof of [5, Lemma 2.2], that $A^{-\alpha / 2}$ is a bounded map from $X_{2}=H_{\sigma}$ to $X_{q}$ provided that $q \leq 2 n /(n-2 \alpha)$. Thus there exists a constant $M_{q}$ such that $\left\|A^{-\alpha / 2} w\right\|_{q} \leq M_{q}\|w\|_{2}$ for all $w \in X_{2}$. Letting $v=A^{-\alpha / 2} w$, we see that for all $v \in D\left(A^{\alpha / 2}\right) \cap X_{2}$

$$
\|v\|_{q} \leq M_{q}\left\|A^{\alpha / 2} v\right\|_{2}
$$


whenever $q \leq 2 n /(n-2 \alpha)$. Since $M_{q}$ comes only from the embedding of $D\left(A^{\alpha / 2}\right)$ into $X_{2} \cap H^{\alpha}(\Omega)$ and from the Sobolev inequalities, the arguments in [1] apply again here to show that the constant $M_{q}$ can be chosen so as to not increase as $\lambda_{1}$ increases, at least for $\alpha \leq 1$.

From basically the same calculation that leads to (2.1) we also see that

$$
\|v\|_{2} \leq \lambda_{1}^{-1 / 2}\left\|A^{1 / 2} v\right\|_{2}
$$

for all $v \in D(A)$ and that

$$
\left\|e^{-t A} v\right\|_{2} \leq\|v\|_{2} e^{-\lambda_{1} t}
$$

for all $v \in L^{2}(\Omega)$ and all $t \geq 0$. For the details of these calculations, see e.g. [1, (2.1) and (2.3)]. The semigroup $e^{-t A}$ is, moreover, analytic on all the $L^{p}$-spaces, $1<p<+\infty([5])$; thus there exists a constant $c_{p}\left(=c_{p}(\alpha)\right)$ such that for each $\alpha>0$

$$
\left\|A^{\alpha} e^{-t A} v\right\|_{p} \leq c_{p} t^{-\alpha}\|v\|_{p}
$$

for all $v \in L^{p}(\Omega)$ and all $t \geq 0$. That $c_{p}$ does not increase as $\lambda_{1}$ increases can be seen from remarks made in $[1, \S 2]$.

We now verify (1.4). Let $v$ be a Leray solution, then, in particular,

$$
\frac{\partial}{\partial t}(v, v)+\left(A^{1 / 2} v, A^{1 / 2} v\right)=(f, v)=\left(A^{-1 / 2} f, A^{1 / 2} v\right),
$$

where we haved used the standard fact that $(v, P(v \cdot \nabla) v)=(P v,(v \cdot \nabla) v)=$ $(v,(v \cdot \nabla) v)=-((\nabla \cdot v) v, v)=0$. Hence

$$
\frac{1}{2} \frac{d}{d t}\|v\|_{2}^{2}+\left\|A^{1 / 2} v\right\|_{2}^{2} \leq \frac{1}{2}\left\|A^{1 / 2} v\right\|_{2}^{2}+\left\|\frac{1}{2} A^{-1 / 2} f\right\|_{2}^{2}
$$

from which we obtain, using (2.3),

$$
\frac{d}{d t}\|v\|_{2}^{2}+\left\|A^{1 / 2} v\right\|_{2}^{2} \leq\left\|\frac{1}{\lambda_{1}^{1 / 2}} f\right\|_{2}^{2} \leq \frac{1}{\lambda_{1}^{1 / 2}} L
$$

where $L$ is as in (1.3). Integrating both sides of (2.8) and dividing by $t$, we have

$$
\frac{1}{t} \int_{0}^{t}\left\|A^{1 / 2} v\right\|_{2}^{2} d s \leq\left\|\frac{1}{t} v_{0}\right\|_{2}^{2}+\frac{1}{\lambda_{1}^{1 / 2}} L
$$

for all $t \geq 0$. Setting $t=T \equiv\left(\lambda_{1}^{1 / 2}\left\|v_{0}\right\|_{2}^{2}\right) /(\delta L)$ for a given $\delta \geq 0$, we thus obtain from (2.9) that

$$
\frac{1}{T} \int_{0}^{T}\left\|A^{1 / 2} v\right\|_{2}^{2} d s \leq \frac{1+\delta}{\lambda_{1}^{1 / 2}} L,
$$

and thus (1.4) is established for some $\tau_{v} \in(0, T)$.

By combining with (2.2) with $q=4$, we thus have that

$$
\left\|v\left(\tau_{v}\right)\right\|_{4} \leq \frac{(1+\delta)^{\frac{1}{2}}}{\lambda_{1}^{1 / 4}} M_{4} L^{\frac{1}{2}} .
$$

We now compare the bound (2.11) with the bound imposed on $u_{0}$ and $f$ in [1] that guarantees existence and uniqueness of global strong solutions. This bound is 
imposed collectively via the quantity $M$ mentioned in the introduction, which in light of (1.3) simplifies to

$$
M \equiv C_{4}\left\|u_{0}\right\|_{4}+3^{3 / 8} c_{2} M_{4} L\left(2 / \lambda_{1}\right)^{5 / 8}\left[8 / 5+e^{-1}\right]
$$

where $L$ is as in (1.3), $c_{2}$ is as in (2.5) with $p=2$, and $C_{4}$ is a constant such that

$$
\left\|e^{-t A} h\right\|_{4} \leq C_{4}\|h\|_{4}
$$

for all $h \in L^{4}(\Omega)$. Basically $M$ is an estimate of

$$
\left\|e^{-t A} u_{0}+\int_{0}^{t} e^{-(t-s) A} f(s) d s\right\|_{4}
$$

for the details of its derivation, see [1, equation (1ML4)] as well as the concluding remarks of [1].

As noted in the introduction, the bound required in [1] on $M$ is

$$
M<\frac{\lambda_{1}{ }^{1 / 8}}{K_{1}}
$$

where $K_{1}$ is a constant depending on $M_{4}, c_{2}$, etc. To see what this requires so that $v$ continues as a string solution, we substitute the right-hand side of (2.11) for $\left\|u_{0}\right\|_{4}$ in (2.12) and then replace $M$ in (2.15) with the resultant right-hand side of (2.12); after some arithmetic, we obtain the condition that

$$
\left(K_{2}+K_{3} \lambda_{1}^{-1 / 8}\right) L \leq \frac{\lambda_{1}^{5 / 8}}{K_{1} M_{4}},
$$

where $K_{2}=C_{4}(1+\delta)$ and $K_{3}=2^{5 / 8} 3^{3 / 8} c_{2}\left[8 / 5+e^{-1}\right]$. Thus we see that if $\Omega$ is generalized thin enough, i.e. if $\lambda_{1}$ is large enough, then (2.16), and hence (2.15), is eventually satisfied; and thus every Leray solution eventually continues as a strong solution; alternatively, if $L$ is small enough in (2.16), the same conclusion holds. In particular these are the conditions on $\lambda_{1}$ referred to in the statement of Corollary 1.2 and the remarks preceding it, and under these conditions we thus obtain (1.4) and (2.11) with $\tau_{v}$ replaced by any $t \geq \tau_{v}$.

We note that the power on $\lambda_{1}$ in (2.16) is significantly larger than the corresponding power in (2.15). This means, even in cases when (2.15) imposes significant size restrictions on initial data in order to guarantee global strong solutions, that nonetheless (2.16) may still be satisfied so that Leray solutions will eventually become regular. This can loosely be characterized as happening on moderatelythin domains with moderately-sized forcing data $f$. We now develop an estimate that will be useful in the next section. For divergence-free $v$ we note the standard observation that each component of $(v \cdot \nabla) v$ can be written as $\nabla \cdot\left(v_{i} v\right)$, so that if we define $\left(v^{\wedge} v\right) \equiv\left(v_{1} v, v_{2} v, v_{3} v\right) \in\left(\mathfrak{R}^{3}\right)^{3}$, we can thus write $A^{-1 / 2} P(v \cdot \nabla) v$ as $T\left(v^{\wedge} v\right)$, where $T \equiv A^{-1 / 2} P d i v$ and it is understood that the $i$ th component of $T\left(v^{\wedge} v\right)$ is $A^{-1 / 2} P \operatorname{div}\left(v_{i} v\right)$. But by [5, Lemma 2.1], $T$ is a bounded operator on $L^{2}(\Omega)$; we set $B \equiv\|T\|_{2}$. Set $\gamma_{3}=\lambda_{1} / 3$; then we are ready to state our needed estimate as a lemma:

Lemma 2.1. Let $v=\left(v_{1}, v_{2}, v_{3}\right)$ be in $V$. Then there exists a constant $K$ such that

$$
\left\|e^{-t A} P \operatorname{div}\left(v^{\wedge} v\right)\right\|_{4}=\left\|e^{-t A} P(v \cdot \nabla) v\right\|_{4} \leq K \frac{e^{-\gamma_{3} t}}{t^{7 / 8}}\left\|v^{\wedge} v\right\|_{2}
$$


Proof. Using (2.2), (2.4), and (2.5), we have that

$$
\begin{aligned}
\left\|e^{-t A} P(v \cdot \nabla) v\right\|_{4} & =\left\|\left(e^{-(1 / 3) t A}\right)^{3} P(v \cdot \nabla) v\right\|_{4} \\
& \leq M_{4}\left\|A^{3 / 8} e^{-(1 / 3) t A}\left(e^{-(1 / 3) t A}\right)^{2} P(v \cdot \nabla) v\right\|_{2} \\
& \leq 3^{3 / 8} c_{2} M_{4} t^{-3 / 8}\left\|e^{-(1 / 3) t A}\left(e^{-(1 / 3) t A} P(v \cdot \nabla) v\right)\right\|_{2} \\
& \leq 3^{3 / 8} c_{2} M_{4} t^{-3 / 8} e^{-\gamma_{3} t}\left\|e^{-(1 / 3) t A} P(v \cdot \nabla) v\right\|_{2} \\
& =3^{3 / 8} c_{2} M_{4} t^{-3 / 8} e^{-\gamma_{3} t}\left\|A^{1 / 2} e^{-(1 / 3) t A} T\left(v^{\wedge} v\right)\right\|_{2} \\
& \leq 3^{7 / 8} c_{2}^{2} M_{4} t^{-7 / 8} e^{-\gamma_{3} t}\left\|T\left(v^{\wedge} v\right)\right\|_{2} \\
& \leq 3^{7 / 8} c_{2}^{2} M_{4} B t^{-7 / 8} e^{-\gamma_{3} t}\left\|v^{\wedge} v\right\|_{2} .
\end{aligned}
$$

Thus the lemma is proven with $K=3^{7 / 8} c_{2}^{2} M_{4} B$. Lemma 2.1 provides the key estimate for the developments in [1] as well as what follows here. We now construct an alternative proof, suggested by the referee, that is less streamlined but has the advantages of avoiding the complex machinery of fractional powers of $A$ and producing a constant $K$ that is more explicit, i.e. $M_{4}$ is replaced by the number $2^{1 / 2}$. This new proof replaces the use of $(2.2)$ with the following estimate, which is a special case of the Gagliardo-Nirenberg estimates; see e.g. [3, Theorem 9.3, and the Bibliographical Remarks, p. 251]:

$$
\|v\|_{4} \leq C\|\nabla v\|_{2}^{3 / 4}\|v\|_{2}^{1 / 4}
$$

for all $v \in H_{0}^{1}(\Omega)$.

In fact, (2.19) also appears in [13, Lemma III.3.5] with $C \equiv 2^{1 / 2}$. Combining this observation with (2.1), we have for all $v \in H_{0}^{1}(\Omega)$ that

$$
\|v\|_{4} \leq 2^{1 / 2}\left\|A^{1 / 2} v\right\|_{2}^{3 / 4}\|v\|_{2}^{1 / 4}
$$

To estimate the left-hand side of (2.17), we now set $\gamma_{12}=\lambda_{1} / 12=\gamma_{3} / 4$ and use (2.20) with (2.4) and (2.5) to obtain

$$
\begin{aligned}
\left\|e^{-t A} P(v \cdot \nabla) v\right\|_{4}= & \left\|\left(e^{-(1 / 3) t A}\right)^{3} P(v \cdot \nabla) v\right\|_{4} \\
\leq & 2^{1 / 2}\left\|A^{1 / 2} e^{-(1 / 3) t A}\left(e^{-(1 / 3) t A}\right)^{2} P(v \cdot \nabla) v\right\|_{2}^{3 / 4} \\
& \times\left\|e^{-(1 / 3) t A}\left(e^{-(1 / 3) t A}\right)^{2} P(v \cdot \nabla) v\right\|_{2}^{1 / 4} \\
\leq & 3^{3 / 8} c_{2}^{3 / 4} t^{-3 / 8} 2^{1 / 2}\left\|\left(e^{-(1 / 3) t A}\right)^{2} P(v \cdot \nabla) v\right\|_{2}^{3 / 4} \\
& \times\left\|\left(e^{-(1 / 3) t A}\right)^{2} P(v \cdot \nabla) v\right\|_{2}^{1 / 4}\left(e^{-\gamma_{3} t}\right)^{1 / 4} \\
= & 3^{3 / 8} c_{2}^{3 / 4} 2^{1 / 2} t^{-3 / 8}\left\|\left(e^{-(1 / 3) t A}\right)^{2} P(v \cdot \nabla) v\right\|_{2} e^{-\gamma_{12} t} \\
\leq & 3^{3 / 8} c_{2}^{3 / 4} 2^{1 / 2} t^{-3 / 8} e^{-\gamma_{12} t} e^{-\gamma_{3} t}\left\|A^{1 / 2} e^{-(1 / 3) t A} T\left(v^{\wedge} v\right)\right\|_{2} \\
\leq & 3^{7 / 8} c_{2}^{7 / 4} 2^{1 / 2} B t^{-7 / 8} e^{-\left(\gamma_{3}+\gamma_{12}\right) t}\left\|\left(v^{\wedge} v\right)\right\|_{2} \cdot
\end{aligned}
$$


Thus the lemma is re-proven with $K=3^{7 / 8} c_{2}^{7 / 4} 2^{1 / 2} B$. We have, in fact, a slightly better exponential decay rate, allowing us to replace $\gamma_{3}$ by $\left(\lambda_{1} / 3+\lambda_{1} / 12\right)$ in what follows, although this will not significantly affect the order of magnitude of our estimates.

With this we conclude our preliminary observations and we are ready to prove our main results.

\section{Proofs of The MAIN THEOREMS}

Let $v$ and $w$ be as in Theorem 1.1. For simplicity we set

$$
M^{\prime} \equiv(1+\delta)^{\frac{1}{2}} \lambda_{1}^{-\frac{1}{4}} L^{\frac{1}{2}} .
$$

Let $T$ be a number to be chosen later and let $s$ and $t$ be such that $0<s \leq t \leq T$. We first note that by the standard variation-of-parameters formula we have

$$
v(t)-w(t) \leq e^{-t A}\left(v_{0}-w_{0}\right)+\int_{0}^{t} e^{-(t-s) A} \operatorname{Pdiv}\left(v^{\wedge} v(s)-w^{\wedge} w(s)\right) d s .
$$

Taking the $L^{4}$-norm of both sides of (3.2) and using Lemma 2.1, we obtain

$$
\|v(t)-w(t)\|_{4} \leq\left\|e^{-t A}\left(v_{0}-w_{0}\right)\right\|_{4}+K \int_{0}^{t} \frac{e^{-\gamma_{3}(t-s)}}{(t-s)^{7 / 8}}\left\|v^{\wedge} v(s)-w^{\wedge} w(s)\right\|_{2} d s .
$$

Now it is clear from the discussion following Theorem 1.1 that we can assume that for all $t \geq 0 v(t)$ and $w(t)$ satisfy (1.4). Combining this with (2.2) with $q=4$ we thus have that

$$
\begin{gathered}
\left\|v^{\wedge} v(s)-w^{\wedge} w(s)\right\|_{2} \leq\|v(s)\|_{4}\|v(s)-w(s)\|_{4}+\|w(s)\|_{4}\|v(s)-w(s)\|_{4} \\
\leq 2 M_{4} M^{\prime}\|v(s)-w(s)\|_{4} .
\end{gathered}
$$

By standard interpolation results there exists a constant $C$ such that

$$
\|h\|_{4} \leq C\|h\|_{6}^{3 / 4}\|h\|_{2}^{1 / 4}
$$

for all $h \in L^{6}(\Omega)$. Combining (1.4), (2.2) with $q=6$, (2.4), and (3.5), we have

$$
\begin{aligned}
\left\|e^{-t A}\left(v_{0}-w_{0}\right)\right\|_{4} \leq & C\left(2 M_{4} M^{\prime}\right)^{3 / 4}\left\|v_{0}-w_{0}\right\|_{2} e^{-\lambda_{1} t} \\
& \equiv C_{0} e^{-\lambda_{1} t} \\
& \leq C_{0} e^{-\gamma_{6} t}
\end{aligned}
$$

where $\gamma_{6}=\lambda_{1} / 6$. We now combine (3.3), (3.4), and (3.6), and set $K_{0}=2 K M_{4} M^{\prime}$, to obtain

$$
\|v(t)-w(t)\|_{4} \leq C_{0} e^{-\gamma_{6} t}+K_{0} e^{-\gamma_{6} t} \int_{0}^{t} \frac{e^{-\gamma_{6}(t-s)}}{(t-s)^{7 / 8}}\left(e^{\gamma_{6} s}\|v(s)-w(s)\|_{4}\right) d s .
$$

Setting $\rho(t)=e^{\gamma_{6} t}\|v(t)-w(t)\|_{4}$ and multiplying both sides of (3.7) by $e^{\gamma_{6} t}$, we have

$$
\rho(t) \leq C_{0}+K_{0} \int_{0}^{t} \frac{e^{-\gamma_{6}(t-s)}}{(t-s)^{7 / 8}} \rho(s) d s
$$


Clearly $\rho \in C[0, T]$; if we choose $t_{*} \in[0, T]$ such that $\rho\left(t_{*}\right)=\sup _{[0, T]} \rho(t)$, then using (3.8) we have

$$
\begin{gathered}
\rho(t) \leq C_{0}+K_{0} \rho\left(t_{*}\right) \int_{0}^{t} \frac{e^{-\gamma_{6}(t-s)}}{(t-s)^{7 / 8}} d s \\
=C_{0}+K_{0} \rho\left(t_{*}\right) \int_{0}^{t} \frac{e^{-\gamma_{6} s}}{s^{7 / 8}} d s \leq C_{0}+K_{0} \rho\left(t_{*}\right) \int_{0}^{\infty} \frac{e^{-\gamma_{6} s}}{s^{7 / 8}} d s \\
\equiv C_{0}+K_{0} \rho\left(t_{*}\right) \delta_{6}\left(\lambda_{1}\right)
\end{gathered}
$$

for all $t \in[0, T]$. Hence

$$
\rho\left(t_{*}\right) \leq C_{0}+K_{0} \delta_{6}\left(\lambda_{1}\right) \rho\left(t_{*}\right)
$$

and thus

$$
\left(1-K_{0} \delta_{6}\right) \rho\left(t_{*}\right) \leq C_{0}
$$

Now clearly $\delta_{6}\left(\lambda_{1}\right) \rightarrow 0$ as $\lambda_{1} \rightarrow \infty$ (see e.g. [1] for explicit details), so choose $\lambda_{1}$ large enough so that $V_{1}\left(\lambda_{1}\right) \equiv 1-K_{0} \delta_{6}\left(\lambda_{1}\right)>0$. Then from (3.11) we have

$$
\rho\left(t_{*}\right) \leq C_{0} V_{1}^{-1} \equiv V_{0}\left(\lambda_{1}\right) .
$$

Since $T$ is arbitrary we thus have from (3.12) that

$$
\|v(t)-w(t)\|_{4} \leq V_{0} e^{-\gamma_{6} t}
$$

for all $t \geq 0$; this proves Theorem 1.1.

We now examine how to modify the above proof so as to obtain Theorem 1.3. To obtain an estimate on $v-w_{1}$, we can use (3.7) with $w_{1}$ in place of $w$, provided that we add the term $\int_{0}^{t}\left\|e^{-(t-s) A}\left(f(s)-f_{1}\right)\right\|_{4} d s$; using (2.2) with $q=4$ and (2.5) with $p=2$, we estimate this term as follows:

$$
\begin{gathered}
\int_{0}^{t}\left\|e^{-(t-s) A}\left(f(s)-f_{1}\right)\right\|_{4} d s \\
\leq M_{4} \int_{0}^{t} \| A^{1 / 2} e^{-[(t-s) / 2] A}\left(e^{-[(t-s) / 2] A}\left(f(s)-f_{1}\right) \|_{2} d s\right. \\
\leq 2^{1 / 2} M_{4} c_{2} \int_{0}^{t}(t-s)^{-1 / 2}\left\|e^{-[(t-s) / 2] A}\left(f(s)-f_{1}\right)\right\|_{2} d s \\
\leq 2^{1 / 2} M_{4} c_{2} \int_{0}^{t}(t-s)^{-1 / 2} e^{-\gamma_{2}(t-s)}\left\|\left(f(s)-f_{1}\right)\right\|_{2} d s \\
=2^{1 / 2} M_{4} c_{2} \int_{0}^{t} s^{-1 / 2} e^{-\gamma_{2} s}\left\|f(t-s)-f_{1}\right\|_{2} d s \\
\leq 2^{1 / 2} M_{4} c_{2} \int_{0}^{\infty} s^{-1 / 2} e^{-\gamma_{2} s}\left\|f(t-s)-f_{1}\right\|_{2} d s,
\end{gathered}
$$

where $\gamma_{2}=\lambda_{1} / 2$. Since the integrand in the last term of (3.14) is dominated by $2 L s^{-1 / 2} e^{-\gamma_{2} s}$ and goes to zero pointwise as $t \rightarrow \infty$, we see that all terms in (3.14) vanish as $t \rightarrow \infty$. In particular the first term can be added to the right-hand sides of all estimates following (3.7) (in some cases multiplied by $e^{-\gamma_{6} t}$ ), until finally it appears as an additional vanishing term on the right-hand side of (3.13). With these observations we obtain Theorem 1.3.

We conclude this section by proving Theorem 1.4. Using the remarks preceding Lemma 2.1, we rewrite (1.9) as

$$
u=A^{-1} f_{1}-A^{-1 / 2} T\left(u^{\wedge} u\right),
$$


which we will now proceed to solve by a contraction mapping argument. Set $M=$ $\left\|A^{-1} f_{1}\right\|_{4}$; then $A^{-1} f_{1} \in L^{4}(\Omega)$ follows easily from (2.2). Set

$$
E=\left\{v \in X_{4}:\left\|v-A^{-1} f_{1}\right\|_{4} \leq M\right\}
$$

and set $S(v)=A^{-1 / 2} T\left(v^{\wedge} v\right)$. We first show that $S$ maps $E$ to $E$ if $\lambda_{1}$ is large enough; in fact, using (2.2) with $q=2$ and $\alpha=3 / 4$, and noting that (2.3) is true for any positive power $p$ replacing $1 / 2$, we have

$$
\begin{gathered}
\left\|S(v)-A^{-1} f_{1}\right\|_{4}=\left\|A^{-1 / 2} T\left(v^{\wedge} v\right)\right\|_{4} \\
\leq M_{4}\left\|A^{3 / 8} A^{-1 / 2} T\left(v^{\wedge} v\right)\right\|_{2}=M_{4}\left\|A^{-1 / 8} T\left(v^{\wedge} v\right)\right\|_{2} \\
\leq M_{4} \lambda_{1}^{-1 / 8}\left\|T\left(v^{\wedge} v\right)\right\|_{2} \leq M_{4} B \lambda_{1}^{-1 / 8}\left\|v^{\wedge} v\right\|_{2} \\
\leq M_{4} B \lambda_{1}^{-1 / 8}\|v\|_{4}^{2} \leq M_{4} B(2 M) \lambda_{1}^{-1 / 8} .
\end{gathered}
$$

By choosing $\lambda_{1}$ large enough, the right hand side of (3.17) can be made to be less than or equal to $M$. A similar argument using the way the term $v^{\wedge} v-w^{\wedge} w$ is handled in (3.4) then shows that for $\lambda_{1}$ large enough $S$ is a contraction on $E$. Thus the existence and uniqueness of the solution $u_{s}$ follows.

To obtain (1.10), we multiply both sides of (1.9) by $u_{s}$ and use arguments similar to those employed in (2.6)-(2.8).

$$
\begin{aligned}
& \left\|A^{1 / 2} u_{s}\right\|_{2}^{2} \leq\left|\left(A^{-1 / 2} f_{1}, A^{1 / 2} u_{s}\right)\right| \\
& \leq \frac{1}{2}\left\|A^{1 / 2} u_{s}\right\|_{2}+\frac{1}{2}\left\|A^{-1 / 2} f_{1}\right\|_{2} .
\end{aligned}
$$

Using (2.3) with $v$ replaced by $A^{-1 / 2} f_{1}$, it is now clear how to obtain (1.10) from (3.18). This completes the proof of Theorem 1.4.

In the spirit of our remarks at the end of section 2 , we now provide an alternative proof of (2.17) that uses (2.19) and (2.3) directly. We have that

$$
\begin{aligned}
\left\|S(v)-A^{-1} f_{1}\right\|_{4} & =\left\|A^{-1 / 2} T\left(v^{\wedge} v\right)\right\|_{4} \\
& \leq 2^{1 / 2}\left\|A^{1 / 2}\left(A^{-1 / 2} T\left(v^{\wedge} v\right)\right)\right\|_{2}^{3 / 4}\left\|A^{-1 / 2} T\left(v^{\wedge} v\right)\right\|_{2}^{1 / 4} \\
& \leq 2^{1 / 2}\left\|T\left(v^{\wedge} v\right)\right\|_{2}^{3 / 4}\left(\lambda_{1}^{-1 / 2}\left\|T\left(v^{\wedge} v\right)\right\|_{2}\right)^{1 / 4} \\
& =2^{1 / 2} \lambda_{1}^{-1 / 8}\left\|T\left(v^{\wedge} v\right)\right\|_{2} \leq 2^{1 / 2} B \lambda_{1}^{-1 / 8}\left\|v^{\wedge} v\right\|_{2} \\
& \leq 2^{1 / 2} B \lambda_{1}^{-1 / 8}\|v\|_{4}^{2} \leq 2^{1 / 2} B(2 M)^{2} \lambda_{1}^{-1 / 8} .
\end{aligned}
$$

We now proceed as before, with $M_{4}$ replaced by $2^{1 / 2}$. With this re-proof of (3.17) we complete the discussions of this section.

\section{REMARKS}

To incorporate $\nu \neq 1$ into our results, we note that this amounts to replacing $M_{q}$ by $\nu^{-\frac{\alpha}{2}} M_{q}$ and $\lambda_{1}$ by $\nu \lambda_{1}$ throughout the paper. Thus the $K$ of Lemma 2.1 would then be replaced by $\nu^{-\frac{3}{8}} K$, according to the proof of that result, and $\delta_{6}$ in the proof of Theorem 1.1 would also have to be appropriately modified, accordingly affecting the rate at which $V_{1}\left(\lambda_{1}\right) \rightarrow 0$ as $\lambda_{1} \rightarrow \infty$.

The end result of these calculations shows that there is a constant $K_{2}$ such that if $M^{\prime}<\nu \lambda_{1}^{\frac{1}{8}} / K_{2}$ then Theorem 1.1 holds. This is similar to the size restriction for 
$\nu \neq 1$ placed on $M$ in [1]; see e.g. the concluding remarks of that paper. In fact the only difference is in the constant $K_{2}$. But this constant is calculated in a fashion very similar to the calculation of $K_{1}$, so that the two constants are very similar in magnitude.

Meanwhile $M$ and $M^{\prime}$ are related by the discussion surrounding (2.16), which, for large $\lambda_{1}$, is, if anything, an easier criteria to fullfil than (2.15) is for the initial and forcing data of global strong solutions. Thus for "most cases" where (1.7) is satisfied, the attractor consists of the single point $u_{s}$. Since the attractor $\mathcal{A}$ still exists, however, in cases when (1.7) is not true, there remains work to be done in further characterizing the attractor in cases where $f$ is e.g. periodic or almost periodic.

\section{ACKNOWLEDGMENTS}

The author would like to thank his visiting colleague A. Babin for suggesting this line of questioning, his colleague B. Vainberg for helpful suggestions regarding the proof of Theorem 1.1, and the referee for helpful suggestions including the alternative arguments based on (2.19).

\section{REFERENCES}

[1] J.D. Avrin, Large-eigenvalue global existence and regularity results for the Navier-Stokes equation, J. Diff. Eqns. 127 (1996), 365-390. MR 97b:35138

[2] C. Foias and G. Prodi, Sur le comportement global des solutions non stationnaires des équations de Navier-Stokes en dimension 2, Rend. Sem. Mat. Univ. Padova 39 (1967), 134. MR 36:6764

[3] A. Friedman, Partial Differential Equations, Holt, Rinehart and Winston, Inc., New York, NY, 1969. MR 56:3433

[4] H. Fujita and T. Kato, On the Navier-Stokes initial value problem I, Arch. Rational Mech. Anal. 16 (1964), 269-315. MR 29:3774

[5] Y. Giga and T. Miyakawa, Solutions in $L_{r}$ of the Navier-Stokes initial value problem, Arch. Rational Mech. Anal. 89 (1985), 267-281. MR 86m:35138

[6] C. Guillopé, Comportement à l'infini des solutions des équations de Navier-Stokes et propriété des ensembles fonctionnels invariants (ou attracteurs), Ann. Inst. Fourier (Grenoble) 32 (1982), 1-37. MR 84f:35116

[7] J.G. Heywood, The Navier-Stokes equations: on the existence, regularity and decay of solutions, Ind. U. Math. J. 29 (1980), 639-681. MR 81k:35131

[8] E. Hopf, Über die Anfangswertaufgabe für die hydrodynamischen Grundgleichungen, Math. Nachr. 4 (1951), 213-231. MR 14:327b

[9] O.A. Ladyzhenskaya, The Mathematical Theory of Viscous Incompressible Flow, 2nd ed. (English translation), Gordon and Breach, New York, 1969. MR 27:5034b (1st ed.)

[10] J. Leray, Etude de diverses équations intégrales nonlinéaires et de quelques problèmes que pose l'hydrodynamique, J. Math. Pures Appl. 12 (1933), 1-82.

[11] G. Raugel and G.R. Sell, Navier-Stokes equations on thin 3D domains I: global attractors and global regularity of solutions, J. Amer. Math. Soc. 6 (1993), 503-568. MR 93j:35134

[12] J. Serrin, On the interior regularity of weak solutions of the Navier-Stokes equations, Arch. Rational Mech. Anal. 9 (1962), 187-195. MR 25:346

[13] R. Témam, Navier-Stokes Equations, North-Holland, Amsterdam, 1977. MR 58:29439

[14] R. Témam, Navier-Stokes Equations and Nonlinear Functional Analysis, CBMS Regional Conference Series, No. 41, SIAM, Philadelphia, 1983. MR 86f:35152

Department of Mathematics, University of North Carolina at Charlotte, CharLOTTE, NorTh CARolina 28223

E-mail address: jdavrin@email.uncc.edu 